Baker Emma (Orcid ID: 0000-0003-1165-0490)

Baker, E. Hyperarousal and Insomnia in ASD

\title{
Assessing a Hyperarousal Hypothesis of Insomnia in Adults with Autism Spectrum Disorder
}

Running title: Hyperarousal and insomnia in ASD

Emma K. Baker ${ }^{1,2,3}(\mathrm{PhD})^{*}$, Amanda L. Richdale ${ }^{1}(\mathrm{PhD})$, Agnes Hazi ${ }^{4}$ (MSc), and Luke A. Prendergast $^{5}(\mathrm{PhD})$

${ }^{1}$ La Trobe University, Olga Tennison Autism Research Centre, School of Psychology and Public Health, Bundoora, VIC, Australia 3086

${ }^{2}$ Diagnosis and Development, Murdoch Children’s Research Institute, Parkville, VIC, Australia 3052

${ }^{3}$ University of Melbourne, Department of Paediatrics, Parkville, VIC, Australia 3052

${ }^{4}$ La Trobe University, School of Psychology and Public Health, Bundoora, VIC, Australia 3086

${ }^{5}$ La Trobe University, Department of Mathematics and Statistics, Bundoora, VIC, Australia 3086

*Dr Baker's current address is as per the correspondence address below.

\section{Corresponding Author:}

Emma K. Baker

Diagnosis and Development

Murdoch Children's Research Institute

50 Flemington Road, Parkville, VIC, 3052

This is the author manuscript accepted for publication and has undergone full peer review but has not been through the copyediting, typesetting, pagination and proofreading process, which may lead to differences between this version and the Version of Record. Please cite this article as doi: 10.1002/aur.2094

This article is protected by copyright. All rights reserved. 
AUSTRALIA

P: +61 383416245

E: emma.baker@mcri.edu.au

This article is protected by copyright. All rights reserved. 
Baker, E. Hyperarousal and Insomnia in ASD

\section{Acknowledgements}

We thank all the participants involved in the study for their valuable contribution. This project received partial support from a PhD grant from the APEX Trust for Autism.

\section{Conflict of Interest}

The authors disclose no conflicts of interest.

This article is protected by copyright. All rights reserved. 
Lay Summary: Both objective (cortisol) and subjective (somatic) physiological arousal were associated with poor sleep quality in adults on the autism spectrum. Adults with ASD who were not medicated for a comorbid diagnosis of anxiety and/or depression also had dampened cortisol secretion, suggesting a dysregulation of the hypothalamic pituitary axis. Longitudinal studies investigating the relationship between sleep, psychopathology symptoms, and physiological arousal in autistic individuals is warranted. 


\begin{abstract}
This study aimed to investigate the relationship between sleep, psychopathology (anxiety, depression and pre-sleep arousal) symptoms, and cortisol in adults with ASD. The sample comprised 29 adults with ASD (51.7\% males) and 29 control adults (51.7\% males) aged 21-44 years. Thirteen adults with ASD were medicated for a comorbid diagnosis of anxiety and/or depression (ASD-Med), while the remaining 16 adults with ASD were not medicated for such diagnoses (ASD-Only). Participants completed a questionnaire battery, 14-day sleep/wake diary and 14-day actigraphy assessment. On one day during the data collection period, participants collected five saliva samples, hourly, prior to sleep and two morning samples; immediately upon waking and 30 minutes thereafter for the analysis of cortisol. Cortisol one hour prior to habitual sleep onset time was associated with poorer sleep efficiency in both ASD groups and increased wake after sleep onset duration (ASD-Only). Higher subjective somatic arousal was also associated with increased sleep onset latency, regardless of group, and poorer sleep efficiency in the ASD-Only group. ASD-Only participants had significantly greater reductions in evening cortisol concentrations compared to both ASD-Med and control participants. No significant group differences were found for the cortisol awakening response. Findings suggest a hyperarousal hypothesis of insomnia in adults with ASD. Moreover, the low cortisol levels observed in ASD-Only adults suggest dysregulation of the hypothalamic-pituitary-adrenal (HPA) axis. Longitudinal studies exploring the interplay between insomnia, anxiety and HPA axis regulation across the lifespan in those with ASD are warranted.
\end{abstract}

Key words: Hyperarousal, Autism, Cortisol, Sleep, Anxiety, Insomnia 


\section{Introduction}

Symptoms of insomnia (e.g., difficulties initiating and maintaining sleep) are frequently reported for individuals with Autism Spectrum Disorder (ASD; Richdale \& Schreck, 2009), though the underlying cause of these sleep problems remains unclear. In the general population insomnia is argued to be a result of a "primary physiological disorder of arousal” (p.g., 97; Bonnet \& Arand, 1997). Research has shown increased electrodermal activity, heart rate and rectal temperature, not only 30 minutes prior to and during sleep (Monroe, 1967), but also across the entire 24-hour period (Broman \& Hetta, 1994), in insomnia patients. Moreover, the most meaningful predictor of poor sleep under situational stress in typical adult sleepers was baseline central nervous system (CNS) arousal (Bonnet \& Arand, 2003). Therefore, individuals with elevated baseline CNS arousal may be more susceptible to developing insomnia. In particular, those with ASD are described as having autonomic hyperarousal (Kushki, Brian, Dupuis, \& Anagnostou, 2014; Kushki et al., 2013) which may be a causative factor in the development of insomnia in this population.

In the general population, further support for the hyperarousal theory comes from findings showing elevated cortisol levels across the 24-hour period in insomnia patients (Rodenbeck, Huether, Ruther, \& Hajak, 2002; Vgontzas et al., 2001). Similar findings have also been reported for those with ASD. Richdale and Prior (1992) observed a trend towards daytime cortisol hypersecretion in 19 children with ASD (IQ Range 58-112), with this effect being more pronounced in older children (> 8 years). Similarly, Corbett and colleagues (2008) found children with ASD had consistently elevated evening cortisol levels and more variable cortisol levels compared to control children. This elevation in cortisol provides support for a hyperarousal hypothesis of insomnia for individuals with ASD. Nonetheless the relationship between sleep, subjective pre-sleep arousal, and cortisol is yet to be systematically investigated in ASD. 
In the general population, elevated evening cortisol levels have been associated with increased wake after sleep onset (WASO) duration in both subjects with insomnia and those without (Rodenbeck et al., 2002). In turn, increased WASO and poorer sleep quality were associated with lower cortisol secretion at awakening in both insomnia patients and controls (Backhaus, Junghanns, \& Hohagen, 2004). Furthermore, lower cortisol awakening response (CAR; Chida \& Steptoe, 2009) was linked with poorer self-reported sleep in health populations (Jackowska, Ronaldson, Brown, \& Steptoe, 2016). The period around sleep onset has also been identified as a key time point for alterations in cortisol secretion in individuals with affective disorder (Dahl et al., 1996; Forbes et al., 2006).

Poor sleep in ASD has been associated with anxiety (Mayes \& Calhoun, 2009) and recent research has demonstrated both parent-reported child sleep anxiety and self-reported pre-sleep arousal are associated with insomnia symptoms (Hollway, Aman, \& Butter, 2013; Richdale, Baker, Short, \& Gradisar, 2014). Nonetheless, a study conducted by Goldman and colleagues (2017), found similar morning and evening cortisol concentrations in a sample of 26 adolescents and young adults with ASD compared to 13 typically developing adolescents and adults, despite the ASD group having significantly poorer sleep onset latency (SoL) by questionnaire and actigraphy. However, this study did not specifically explore the relationships between actigraphic sleep parameters and cortisol concentrations. Only one publication, a conference abstract (Chicoine et al., 2013), has examined the relationship between sleep and salivary cortisol in 13 individuals with ASD $\left(M_{\text {age }}=22.2, S D=3.7\right.$ years) compared to 12 control adults ( $M_{\mathrm{age}}=21.8, S D=4.2$ years). A positive correlation between evening cortisol levels and the number and duration of nocturnal awakenings and a negative correlation between evening cortisol and sleep efficiency (SE\%; ratio of total sleep time compared to total time in bed) was seen in the control group, but not for the adults with ASD. However, a negative correlation between cortisol and Stage 4 slow-wave sleep duration was observed for the ASD group. 
Thus, emerging evidence suggests that insomnia in individuals with ASD is associated with anxiety and hyperarousal, but further research is needed. The aims of this study were (i) to assess subjective psychopathology symptoms (anxiety and depression), pre-sleep arousal (somatic and cognitive) and salivary cortisol (morning, evening) in adults with ASD and no comorbid intellectual disability compared to sex- and IQ-matched controls; and (ii) to explore relationships between psychopathology symptoms, cortisol levels and objectively measured sleep parameters in both groups. It was hypothesized that adults with ASD would have higher evening cortisol concentrations and lower awakening cortisol concentrations compared to controls. It was also hypothesized that evening cortisol concentrations would be positively correlated with SoL and WASO and negatively associated with SE\% and total sleep time (TST) in both groups of adults, while opposite effects would be observed for the cortisol awakening response (CAR).

\section{Methods}

\section{Participants}

Participants included 29 adults with a clinical diagnosis of ASD (51.7\% male) and 29 healthy control adults (51.7\% male). Thirteen participants with ASD reported a comorbid clinical diagnosis of an anxiety and/or mood disorder and were taking associated medications (ASD-Med); four had depression, four had an anxiety disorder and five had both. Thus, two ASD groups are included here; ASD-Only (56.3\% male) and ASD-Med (46.2\% male). The three groups did not differ on the proportion of males in each group, $\chi^{2}=.293, p=.864, \Phi=.071$ and were also matched on age, full scale IQ (FSIQ) and Body Mass Index (BMI; see Table 1). Although a greater proportion of control adults were using an oral contraceptive or Implanon ${ }^{\circledR}$, the three groups did not significantly differ on the proportion of those using such contraception, Likelihood ratio $=1.375, p=.503, \Phi=.163$ (see Table S1 for details of medications). No participants in any group reported having ever received a diagnosis of Attention Deficit Hyperactive Disorder. 
ASD participants were primarily recruited through autism associations and support groups while control participants were primarily recruited through a university student participant registry and flyers placed around the university campus. The recruitment flyer for both groups stated that participants were being sought for a 'sleep study' and in bold text "You DO NOT need to have a sleep problem to participate”. For further details, see Baker and Richdale (2015).

The Wechsler Abbreviated Scale of Intelligence (WASI; Wechsler, 1999) was administered to 24 ASD and 28 control participants. Five ASD participants had recently completed the Wechsler Adult Intelligence Scale - Fourth Edition (WAIS-IV; Wechsler, 2008) and scores were obtained from their reports. One control participant was a professional with a PhD qualification who regularly administered the WASI, thus an assessment would not be valid, and IQ>80 could be assumed. All participants assessed had a FSIQ>80, and the three groups did not differ (Table 1).

\section{Confirming ASD diagnosis}

All ASD participants completed Module 4 of the Autism Diagnostic Observation Schedule $2^{\text {nd }}$ Edition (ADOS-2; Lord et al., 2012). Additionally, 26 participants provided a copy of their diagnostic report and one provided a statement from their clinician confirming their diagnosis; those two participants who did provide diagnostic reports met criteria for ASD on the ADOS-2. All control participants completed the Autism Quotient (AQ; Baron-Cohen et al., 2001) and scored below the clinical cut-off of 26 (Woodbury-Smith, Robinson, Wheelwright, \& Baron-Cohen, 2005). Table 1 provides information regarding age, FSIQ, AQ and ADOS scores for the three groups.

\section{Exclusionary Criteria}

For both ASD and control groups, participants were excluded if they had a diagnosis of schizophrenia or if they were taking any sleep medication. Participants were also excluded from the control group, if they had a diagnosis of an anxiety or mood disorder and/or if they had a first degree relative with a diagnosis of ASD. We had planned to exclude those adults with ASD who were 
medicated for a diagnosis of anxiety and/or depression given the impact such medications may have on cortisol levels. However, given the heightened prevalence of comorbid anxiety and depression reported for adults with ASD (Buck et al., 2014) and the significant number of individuals interested in participating in the study who had such diagnoses, we included these individuals and analysed them as a separate group. Further details regarding exclusionary criteria can be found elsewhere (Baker \& Richdale, 2015).

\section{Sleep Measures}

Participants wore an actigraph monitor (Phillips Respironics 2) on their non-dominant wrist for the 14-day diary period. Data were digitized in 1-minute epochs with a sensitivity of $0.025 \mathrm{~g}$ and a bandwidth of 0.35-7.5 Hz, and analysed with Respironics Actiware 6 software; time in bed (TIB), total sleep time (TST), sleep onset latency (SoL), sleep efficiency (SE\%), and wake after sleep onset (WASO) were extracted. Sleep efficiency is defined as the ratio of TST to total TIB (i.e., [TST / TIB] $\times 100$ ). Sleep onset was defined as the first of five consecutive epochs of actigraphic sleep at the beginning of the scoring interval. Sleep offset was defined as the last of at least five consecutive epochs of actigraphic sleep at the end of the scoring interval. Actigraphy data were verified with a 14day sleep-wake diary; details are reported elsewhere (Baker \& Richdale, 2015).

Participants also completed the Pittsburgh Sleep Quality Index (PSQI; Buysse et al., 1989) as a measure of insomnia symptoms and severity. The PSQI is a self-report measure of sleep quality that was originally developed on two sleep disorder groups, those with major depressive disorder and a specific sleep disorder group, in addition to a sample of good sleepers. The sleep disorder sample comprised patients presenting with difficulties initiating or maintaining sleep and/or excessive daytime sleepiness and had been referred to a sleep disorder clinic. A cut-off score of $>5$ indicates that the individual has severe difficulties in at least two domains or moderate difficulties in more than 
3. The PSQI has demonstrated high test-retest reliability and good validity in patients with primary insomnia when using a cut-off $>5$ (Backhaus, Junghanns, Broocks, Riemann, \& Hohagen, 2002).

\section{Psychopathology Measures}

Participants completed several questionnaires at their convenience during the 14-day data collection period (see Baker and Richdale for further details). The 8-item Patient Health Questionnaire-8 Depression Scale (PHQ-8; Kroenke et al., 2009), which is based on the diagnostic criteria for major depressive disorder (American Psychiatric Association, 2000) was used to assess depression symptoms. In the current study, the PHQ-8 demonstrated good-excellent internal consistency in all three groups (ASD-Only $\alpha=.88$; ASD-Med $\alpha=.88$; Control $\alpha=.71$ ).

The Trait Anxiety subscale from the State-Trait Anxiety Inventory (STAI-T; Spielberger et al., 1983) was used to assess general anxiety symptoms. In the current study, this scale demonstrated excellent internal consistency in all three groups (ASD-Only $\alpha=.94$; ASD-Med $\alpha=.92$; Control $\alpha=$ $.94)$.

The 10-item Sleep Anticipatory Anxiety Questionnaire (SAAQ; Bootzin et al., 1994) was used to assess subjective arousal prior to sleep. Two sub-scale scores measuring self-reported cognitive (SAA-C) and somatic (SAA-S) pre-sleep arousal were calculated. In a sample of adolescents with ASD the SAAQ demonstrated excellent internal consistency ( $\alpha=.92$; Richdale et al., 2014) . In the current study, each scale demonstrated excellent internal consistency for all groups, (ASD-Only $\alpha=.95$; ASD-Med $\alpha=.80$; Control $\alpha=.83$ ).

\section{Cortisol}

Participants were provided with a saliva collection kit, which included seven (five evening, two morning) SalivaBio Oral swabs (SOS; Salimetrics LLC, PA 16803 USA) in collection tubes. The collection tubes were colour coded for ease of sampling. Participants were also provided with a saliva 
collection sheet, which included both written and pictorial instructions for collecting saliva and observed a saliva collection demonstration at their first appointment.

Prior to analysis saliva samples were defrosted and spun at $3000 \mathrm{rpm}$ for 15 minutes to remove particulate matter that may interfere with the assay. For each sample cortisol concentration was determined in duplicate using a commercially available Cortisol Enzyme Immunoassay Kit (EIA Item No. 1-3002; Salimetrics Inc., State College, PA). Optical density of the well was measured at $450 \mathrm{~nm}$ with a synergy HT Multi-detection Microplate Reader (Bio-Tek Instruments Inc., Winoski, VT, USA). Cortisol concentrations were calculated using KC4 v.3.4 Software (Bio-Tek Instruments Inc.). The intra- and inter-assay precision of the cortisol kit was 3.35\% and 3.75\%, respectively, and the detection limit of the assay was $.07 \mu \mathrm{g} / \mathrm{L}$.

\section{Procedure}

Institutional (La Trobe University) ethics approval was granted (Approval \#HEC12-018) and all participants provided written informed consent prior to commencing participation. Participants were instructed to complete their diary every morning and evening for two weeks, wear the actigraphy monitor at all times, except when it may get wet (e.g., showering, swimming etc.) and complete their online questionnaires at their convenience during this period. After participants had completed 7-days of the sleep-wake diary each individual's average habitual sleep onset time was calculated and he/she received a saliva collection protocol sheet detailing individualized sampling times. Evening saliva sampling followed a standard dim light melatonin onset (DLMO) protocol (Benloucif et al., 2008). Thus samples were collected hourly, commencing three hours prior to habitual sleep onset time and ceasing one hour past habitual sleep onset time, and morning sampling was on waking and 30 minutes after (see Figure 1). Collected samples were analyzed for both melatonin (Baker, Richdale, Hazi, \& Prendergast, 2017) and cortisol. To reduce participant burden, only one night of saliva collection was required to be undertaken during the 14-day protocol, rather than two separate collections for each 
hormone. It has been shown that dim light has little effect on cortisol levels whereas bright light has an acute suppressive effect (Jung et al., 2010). On the day of saliva collection participants had to refrain from smoking, and drinking alcohol and caffeine from midday onwards (Limoges, Mottron, Bolduc, Berthiaume, \& Godbout, 2005). Participants were instructed to rinse their mouths out with water only, 10 minutes prior to each evening sample. Participants froze each sample immediately after collection, placing them in their domestic freezer for collection at the end of the two-week datacollection period. Samples were then stored in a university freezer at $-20^{\circ} \mathrm{C}$ until assayed.

[Insert Figure 1 here]

\section{Data Analysis \\ Questionnaire and Sleep Measures}

All psychopathology variables were normally distributed in the ASD-Only group. Somatic arousal scores were not normally distributed in the ASD-Med and Control groups. Two participants in both the ASD-Med and control groups were outliers on somatic arousal; but inspection of the $5 \%$ trimmed means indicated that they did not have a significant impact on the mean. TST was normally distributed in all three groups. SoL was not normally distributed in the ASD-Med and Control groups, while SE\% was not normally distributed in the ASD-Med group and WASO was not normally distributed in ASD-Only group. One control participant and one ASD-Med participant were outliers on SoL and SE\%, respectively, and one ASD-Only and two controls were outliers on WASO. Similar to the questionnaire measures inspection of the $5 \%$ trimmed means indicated that they did not have a significant impact on the mean. Thus, one-way between groups analysis of variance (ANOVA) was used for comparison on variables that were normally distributed across the three groups. Where the assumptions of normality were violated in any group, the Kruskal-Wallis Test was used.

\section{Evening Cortisol}

This article is protected by copyright. All rights reserved. 
Twenty-eight ASD (12 ASD-Med) and 29 control participants provided evening saliva samples, but three ASD-Med, two ASD-Only and two control participants did not adhere to the evening saliva collection protocol: they either did not adhere to the timing of collection or consumed caffeine within 6 hours of commencing sampling. Calculation based on the half-life of caffeine indicates that caffeine would not have been eliminated from their system (Benowitz, 1990). Thus, for analysis, samples were reduced to 14 ASD-Only, 9 ASD-Med and 27 control participants.

The majority of participants in the ASD-Med (55.6\%) and control groups (66.7\%) collected saliva on a weekday, while the majority of participants in the ASD-Only group completed the collection on a weekend (64.3\%), but this group difference was not significant, $\chi^{2}=3.586, p=.166$, $\Phi$ $=.268$.

Multiple linear regression analysis was used to model sleep outcomes on cortisol (time-point 3; sample 3 collected one hour prior to habitual sleep onset time [see Figure 1]) and psychopathology variables, all interacting with group to adjust for differences between groups. Time-point 3 was chosen since it was the strongest correlating cortisol measure with TST, SE\% and SoL. Stepwise variable selection using the Akaike Information Criterion (AIC; Fahrmeir, Kneib, Lang, \& Marx, 2013) was then used to reduce the model and the variables chosen in the model are reported. Initial regression analyses revealed model violations due to skewed evening cortisol. The log transformation was therefore used and results reported as percentage change on the original cortisol scale.

\section{Morning Cortisol}

Twenty-nine adults with ASD (13 ASD-Med) and 29 control participants provided morning saliva samples. Nine participants (two ASD-Only, four ASD-Med, three control) did not collect both saliva samples within 45 minutes of waking (Stalder et al., 2016) and one ASD-Only participant appeared to have blood in their morning saliva samples, and they were therefore excluded from analyses. Thus, the groups comprised 13 ASD-Only, 9 ASD-Med and 26 control participants. 
Analysis of the CAR (cortisol time 2 - cortisol time 1) was carried out using multiple linear regression to compare groups and to adjust for covariates. Given morning cortisol concentrations were not skewed, non-transformed cortisol was used. Stepwise variable selection using the AIC (Fahrmeir et al., 2013) was used to reduce the model.

False Discovery Rate (FDR) using the Benjamini-Hochberg procedure (Benjamini \& Hochberg, 1995) was applied to adjust for multiple comparisons. Corrected significance levels derived from (Weinkauf, 2012) are reported below each table and p-values that remain significant after FDR are bolded.

\section{Results}

Sleep

A significant difference was found between the three groups on PSQI global scores, with Tukey post-hoc analyses revealing that both ASD groups had significantly higher scores compared to controls. The two ASD groups did not significantly differ on PSQI global scores (Table 2). Using the cut-off of $>5$ on the PSQI, seven (77.8\%) and eight (57.1\%) of the ASD-Med and ASD-Only groups, respectively, were poor sleepers compared to nine (33.3\%) controls.

Kruskal-Wallis analyses to compare SoL, WASO and SE\% on the night of saliva collection showed no significant differences between the three groups (Table 2). A One-way between groups ANOVA with Tukey post-hoc tests showed both ASD groups had significantly shorter TST compared to Controls (Table 2).

[Insert Table 2 here]

\section{Psychopathology}

One-way between group ANOVAs with Tukey post-hoc tests showed that both ASD groups had significantly higher scores on all psychopathology measures compared to the control group with 
medium to large effect sizes. The two ASD groups did not differ significantly on any psychopathology variables (Table 3).

[Insert Table 3 here]

\section{Evening Cortisol Comparisons}

As log cortisol was used in the analyses, findings have been converted back to the original scale and reported as percentage change in means. Mean cortisol levels at time-point 1 (baseline, 3 hours prior to habitual sleep time) did not differ between the three groups, and there were no significant group differences in mean cortisol levels at any later time points when compared to baseline levels for the control and ASD-Med groups. However, a linear mixed effect analysis to model repeated measures of log cortisol over time revealed reductions in mean cortisol levels from baseline for the ASD-Only group 1 hour (31.54\% reduction, 95\% Confidence Interval $(\mathrm{CI})=[6.08$, 50.1]), 2 hours (37.47\% reduction, 95\% CI = [6.14, 58.35]), 3 hours $(51.07 \%$ reduction, $95 \% \mathrm{CI}=$ [22.86, 68.97]) and 4 hours (65.93\% reduction, 95\% CI = [44.69, 79.01]) later, see Figure 2.

[Insert Figure 2 here]

\section{Evening Cortisol and Sleep}

Sleep Onset Latency (SoL)

Only somatic arousal and depression scores were retained in the model, which explained 15\% $\left(\mathrm{R}^{2}\right)$ of the variance in SoL (Adjusted $\mathrm{R}^{2}=12 \%$ ). A positive correlation between SoL and somatic arousal was observed, regardless of group $(p=.006)$ with an estimated 1.902 mean increase in SoL per unit increase in somatic arousal (95\% CI [0.585, 3.220]). Although depression scores were retained in the final model, they were not a significant contributor $(p=.106$, estimate $-0.496,95 \%$ CI $[-1.100,0.109])$.

Wake After Sleep Onset (WASO) 
Cortisol, somatic arousal, depression scores, and group interactions were retained in the model, which accounted for $46 \%\left(\mathrm{R}^{2}\right)$ of the variability in WASO (Adjusted $\mathrm{R}^{2}=31 \%$ ). Elevated cortisol levels in the ASD-Only group were associated with increased WASO; however, this was not the case for the ASD-Med or control groups (Table 4).

[Insert Table 4 here]

Overall, in the ASD-Med group, somatic arousal was negatively associated with WASO and depression scores were positively associated. There were no significant associations for somatic arousal and depression in the other two groups.

\section{Sleep Efficiency (SE\%)}

Cortisol, somatic arousal, trait anxiety and group interactions for cortisol and somatic arousal were retained in the model, which explained $68 \%\left(\mathrm{R}^{2}\right)$ of the variability in SE\% (Adjusted $\mathrm{R}^{2}=61 \%$ ). Cortisol was negatively associated with SE\% in the ASD-Only and ASD-Med groups but not in the controls. Higher somatic arousal scores were associated with lower SE\% in the ASD-only group (Table 5). While trait anxiety was retained as a predictor in the model, it was not significant after adjusting for the other variables.

Total Sleep Time (TST)

Cortisol, depression, somatic arousal, trait anxiety, and group interactions for cortisol and trait anxiety were retained in the model, which explained $53 \%\left(\mathrm{R}^{2}\right)$ of the variability in TST (Adjusted $\mathrm{R}^{2}$ $=41 \%$ ). Cortisol was not significantly associated with TST in the ASD-Only or controls groups, but it was in the ASD-Med group with higher cortisol associated with shorter TST. Participants with higher somatic arousal scores had reduced TST, regardless of group. Both Trait anxiety and depression were included as predictors in the model, but were not significant after adjusting for the other variables (Table 6).

\section{Morning Cortisol Comparisons}


An ANOVA comparing the CAR indicated no significant differences between the three groups ( $p=.958$; see Figure 3).

[Insert Figure 3 here]

\section{Morning Cortisol and Sleep}

SoL, SE\%, TST and group interactions were retained in the model, however, the final model provided a poor fit for modelling CAR $\left(\mathrm{R}^{2}=37.0 \%\right.$, adjusted $\left.\mathrm{R}^{2}=18.0 \%\right)$. Significant associations between CAR and sleep parameters were only found for the ASD-Med group (change in CAR per unit of TST: 0.039, [0.014, 0.064], $p=0.003$; change in CAR per unit of SE\%: 0.544, [0.212, 0.876], $p=.002$; change in CAR per unit of SoL: 0.490, [0.222, 0.758], $p=.001$ ).

\section{Morning Cortisol and Psychopathology}

All psychopathology variables were retained in the model. Regardless of group, higher cognitive arousal scores were associated with a greater CAR (0.177 rise in cortisol change per unit change in cognitive arousal, 95\% CI = [0.005, 0.349], $p=.044)$. In the ASD-Med group higher depression scores were associated with a smaller CAR (0.454 reduction in cortisol change per unit change in depression, $95 \% \mathrm{CI}=[-0.748,-0.160, p=.003)$, while in the ASD-Only group higher trait anxiety was associated with a smaller CAR (0.140 reduction in cortisol change per unit change in trait anxiety, 95\% CI $=[-0.238,-0.043], p=.006)$.

\section{Summary of Results}

Overall the findings demonstrate that both groups of adults with ASD had significant symptoms associated with insomnia, as measured by the PSQI, as well as elevated symptoms of psychopathology compared to controls. ASD-Med and control participants had similar cortisol concentrations across all five evening time-points, while ASD-only participants had significant reductions in cortisol from baseline onwards. Despite these significant reductions in cortisol in the ASD-only group, elevated cortisol levels one-hour prior to habitual sleep time, were associated with 
increased WASO and poorer SE\% in this group. For the ASD-Med group elevated cortisol levels onehour prior to habitual sleep time were associated with poorer SE\% and reductions in TST. Evening cortisol levels were not associated with any of the sleep parameters in the control group. Somatic arousal was also significantly associated with several sleep variables. Regardless of group, elevated somatic arousal was associated with increased SoL and reduced TST. While in the ASD-only group somatic arousal was also associated with poorer SE\%. Increased somatic arousal was associated with reduced WASO in the ASD-Med group. Although trait anxiety and depression scores were often retained in the models, they were not significant unique contributors with the exception of increased depression scores associated with increased WASO in the ASD-Med group.

The CAR did not significantly differ between the three groups and sleep parameters were poor predictors of the CAR; however, psychopathology variables were better predictors of the CAR. Regardless of group, higher cognitive arousal scores were associated with a greater CAR. In the ASDMed group higher depression scores were associated with a smaller CAR, as were anxiety scores in the ASD-Only group.

\section{Discussion}

This study aimed to examine a hyperarousal hypothesis of insomnia in adults with ASD and no intellectual impairment. To this end, cortisol profiles on one evening and the following morning were initially examined in ASD-Only, ASD-Med and an age-, sex- and IQ-matched control group. Both groups of adults with ASD presented with significantly elevated scores on the PSQI, indicating moderate to severe symptoms associated with insomnia. Although all three groups had similar baseline evening cortisol concentrations (3 hours prior to habitual sleep onset time), ASD-Only adults had significant decreases in cortisol concentration over the four remaining time points, suggesting a dampening of cortisol secretion in this group. In contrast to the ASD-Med and control groups, the ASD-Only group had a sharp decrease in cortisol concentrations between habitual sleep onset time 
and their actual sleep time on the saliva collection night, but the ASD-Med and control groups both had a slight increase in cortisol concentrations. These findings suggest that in the two latter groups, staying up one hour past habitual sleep time was an acute mild stressor but, either this was not perceived as a stressor by the ASD-Only participants, or their HPA axis failed to respond appropriately. Moreover, morning cortisol concentrations were lower in the ASD-Only group, though the CAR did not differ between the three groups. This reduction in cortisol concentrations in the ASD-Only adults suggests dampened cortisol secretion, a sign of a dysregulated HPA axis (Heim, Ehlert, \& Hellhammer, 2000).

Dieleman and colleagues (2015) proposed that acute stress with associated anxiety leads to hypersecretion of cortisol, however when anxiety becomes chronic, compensatory mechanisms are activated resulting in diminished cortisol secretion with consequent hypocortisolism. The early development and persistence of anxiety and sleep problems in children with ASD (Humphreys et al., 2014; Strang et al., 2012) may lead to over-activation of the HPA axis, with subsequent burnout of the system by adolescence/adulthood, particularly if anxiety and insomnia are left untreated. Of note, our adults with ASD had significantly elevated symptoms of psychopathology, with both non-medicated and medicated groups having average trait anxiety well above the Australian average (Crawford, Cayley, Lovibond, Wilson, \& Hartley, 2011). Thus, despite elevated anxiety symptoms and an anxiety disorder diagnosis for most members in the ASD-Med group, medication appears to have negated the physiological effects of chronic anxiety that can lead to hypocortisolism. Taken together, these findings suggest that chronic anxiety and sleep problems may lead to burnout and hypocortisolism when these problems remain untreated. However, given the cross-sectional nature of this study, longitudinal studies that track sleep, psychopathology and other factors that may contribute towards hypocortisolism from childhood to adolescence, would be beneficial. 
Some research reports in children with ASD also suggest attenuation of the cortisol response with increasing age, though these findings are inconsistent. In a large study of 113 adolescents (7-17 years) with ASD, hypersecretion of cortisol was observed in the evening, but not at any other timepoint in comparison to age- and sex-matched controls. However, the diurnal slope of the ASD group was also blunted (Muscatello \& Corbett, 2018). Both age and puberty were significant predictors of evening cortisol levels, with increased age and pubertal status being associated with higher cortisol levels (Muscatello \& Corbett, 2018). Corbett et al. (2008) and Richdale and Prior (1992) also demonstrated hypersecretion in children with ASD aged 6-12 and 4-14 years, respectively. Tordjman and colleagues (2014) also found hypersecretion, but the slopes of both day and night-time cortisol were flatter in older children (11.3 \pm 4.1 years $)$ with ASD compared to age- and sex-matched controls. Two studies with older children and adolescent males with ASD found evidence for hypocortisolism, particularly in those with elevated anxiety symptoms (Bitsika, Sharpley, Sweeney, \& McFarlane, 2014; Hollocks, Howlin, Papadopoulos, Khondoker, \& Simonoff, 2014). Consistent with the negative association between trait anxiety and CAR in the ASD-Only group in the current study, awakening cortisol levels were inversely associated with self-rated anxiety symptoms in non-medicated 7-16 year-old males with ASD (Bitsika et al., 2014). Non-medicated males with ASD (10-16 years) meeting criteria for an anxiety disorder also had an attenuated cortisol and heart rate response in relation to a psychosocial stressor compared to controls (Hollocks et al., 2014). Moreover, those children with ASD not meeting criteria for anxiety disorder demonstrated a trend for a decreased cortisol response compared to controls, and their scores on the Spence Children's Anxiety Scale (Parent and Child versions; Spence, 1998) were significantly elevated compared to controls (Hollocks et al., 2014).

However, some research studies have found no associations between cortisol and measures of psychopathology, including state anxiety (Simon \& Corbett, 2013). Differences between studies are 
likely due to the paradigm being used as well as the age of the participants. Specifically, the majority of research in individuals with ASD has explored the relationship between cortisol and psychopathology symptoms in relation to a stressor, rather than baseline levels. Edmiston, Blain, and Corbett (2017) have suggested that children with ASD may have limited insight into reporting their physiological arousal "in the moment”, though reporting on stable trait anxiety may be more reliable. Moreover, research in this area has predominantly focused on children and adolescents, with less literature focused on adults with ASD, and developmental effects seem likely. Nevertheless, detection and treatment of both sleep and anxiety disorders in individuals with ASD should be a clinical focus from a young age.

Despite a general dampening of cortisol secretion in ASD-Only participants, both physiological (cortisol) and subjective pre-sleep somatic arousal were associated with poor sleep in adults with ASD; however, associations varied across the ASD-Only and ASD-Med groups. Consistent with the literature in non-autistic patients with insomnia (Rodenbeck et al., 2002), elevated cortisol one hour prior to habitual sleep resulted in poorer SE\% (both ASD groups), increased WASO (ASD-Only) and reduced TST (ASD-Med). Thus, while the ASD-Only group had lower cortisol concentrations generally, individuals within this group with higher cortisol secretion one hour prior to habitual sleep onset had greater WASO and poorer sleep efficiency. Those in the ASD-Med group may have been more likely to perceive the saliva collection (evening and the morning) as a stressor, which is supported by the increase in cortisol between the last two evening samples in this group. In particular, four of the nine (44\%) ASD-Med group participants woke up a minimum of 17-min (maximum 60-min) prior to the instructed morning cortisol collection time; this collection time was already 10 minutes prior to their habitual wake time. Thus, TST was significantly reduced in these participants and may explain the significant associations between cortisol and TST in this particular group. 
Moreover, subjective somatic arousal was the most prominent psychopathology predictor of poor sleep across groups. Higher somatic arousal was significantly associated with increased SoL and there was a trend towards reduced TST for those with higher somatic arousal, regardless of group. Furthermore, in the ASD-Only group increased somatic arousal was associated with reduced SE\%, but not in the ASD-Med group. In contrast increased somatic arousal was associated with reductions in WASO in the ASD-Med group, despite similar somatic arousal scores between the two ASD groups. The medications taken by ASD-Med participants may explain the differences observed here. One of the most commonly reported side effects of antidepressants is drowsiness (Cascade, Kalali, \& Kennedy, 2009). In a rat model, standard antidepressants have been shown to reduce the spontaneous and sensory-evoked firing rate of neurons in the noradrenergic locus coeruleus (West, Ritchie, BossWilliams, \& Weiss, 2009) which plays an essential role in arousal regulation. Thus the medications may assist those ASD-Med individuals with increased somatic arousal to return to sleep more easily if they wake. In line with the literature in those with psychiatric disorders, including depression (Benca, Obermeyer, Thisted, \& Gillin, 1992), increased depression scores were associated with greater WASO in the ASD-Med group. Overall, caution should be used when considering these findings in the ASD-Med group given the small sample size. Future research is required to confirm and expand on these findings.

Given core ASD symptoms, individuals with ASD may be more attuned to somatic arousal. Parents of children with ASD commented on various aspects of sensory stimulation their children experienced while in bed including hearing noises more prominently (including their own physiological noises such as their heartbeat) and refusing to have bedding in contact with their body (Wiggs \& Stores, 2004). Similarly, Mazurek and Petroski (2015) found that both anxiety and sensory over-responsivity predicted poor sleep in a large sample of children and adolescents aged 2-17 years with ASD. Studies in the general population have also indicated that highly anxious adults have an 
increased perception of physiological sensations such as increased perceived arousal despite the absence of actual differences on physiological measures of arousal (Hoehn-Saric \& McLeod, 2000) and this may be particularly true of the adults with ASD in this study.

\section{Limitations}

While this study has provided significant insights into the relationship between hyperarousal and sleep parameters in adults with ASD, several limitations exist. Although it was expected that both evening cortisol and psychopathology symptoms would be positively associated with SoL, the overall fit of the model was poor and cortisol was not retained in the model. This is likely due to the saliva collection protocol setting bedtime one hour past habitual sleep time, as per a standard DLMO protocol. Thus, participants may be sleepier than usual at bedtime, leading to reduced SoL. Supporting this, SoL for the saliva collection night (Table 2) was significantly reduced in each group compared to their average for the remaining 13 days of actigraphy (Baker \& Richdale, 2015). This likely reduced the ability to detect associations between SoL and the subjective arousal measures and cortisol levels. To better clarify the relationship between physiological arousal and SoL, future research assessing arousal prior to sleep should cease sampling at habitual sleep time. Multiple days of sampling may be beneficial in future studies given that significant variability has been observed in previous studies of children with ASD (Tomarken, Han, \& Corbett, 2015) and in the cortisol profiles of the adults included in this study. However, Richdale and Prior (1992) found no significant day effect in children with autism when examining urinary cortisol over two consecutive days. Furthermore, in their recent review, Sharpley, Bitsika, Agnew, and Andronicos (2017) have argued that there is relative stability in cortisol levels across days in children with ASD, that repeated sampling is unrealistically demanding, and that variability may not represent error but rather changes in biological responsiveness to environmental stimuli. Given that differences in cortisol concentrations may exist between weekend and weekday sampling, a consistent sampling day may be 
more important (Kunz-Ebrecht, Kirschbaum, Marmot, \& Steptoe, 2004). However, differences in weekday and weekend cortisol appear to be associated with employment (Karlamangla, Friedman, Seeman, Stawksi, \& Almeida, 2013); given the high rate of unemployment in ASD (e.g., 26.7\% of ASD-Only and $30.8 \%$ of ASD-Med in the current sample), this may be difficult to control in adults with ASD.

Secondly, comorbid diagnoses of anxiety and depression in the ASD-Med group were not confirmed with clinical interviews. Diagnoses were based on self-report and reported use of associated medications for these diagnoses. Given the high prevalence of psychopathology disorders in adults with ASD (Buck et al., 2014), research should continue to include these individuals; however, confirmation of such diagnoses would be beneficial for future research projects. Moreover, given the conflicting findings in the general population regarding the impact of anxiety and depression on cortisol levels (Feder et al., 2004; Heim et al., 2000) future research should aim to recruit larger samples of adults with ASD with comorbid anxiety only and depression only to start to tease apart these findings.

The use of other medications was not controlled in this study and may have influenced cortisol concentrations (Granger, Hibel, Fortunato, \& Kapelewski, 2009). In particular, six participants (2 ASD-Only, 3 ASD-Med, and 1 control) reported regularly taking asthma medications (ß-2 adrenergic agonists - albuterol and formoterol; corticosteroid - budesonide). Exogenous glucocorticoid use within a preceding two-week period has been reported to decrease cortisol concentrations 30-min post-waking on the day of saliva collection, but not at 12 hours post waking (Masharani et al., 2005). Conversely, Shin et al. (2014) found no effect on morning cortisol levels for those whose asthma was well controlled by inhaled corticosteroids. Thus, asthma medication use may have decreased evening cortisol concentrations or the CAR in some participants. 
Although we attempted to collect self-reports of menstrual cycle from the female participants in the current study, many participants had difficultly completing this questionnaire, with missing data for $13.3 \%$ of controls and $14.3 \%$ of the ASD-Only females. Further, $75 \%$ of those in the ASD-Med group and $42.9 \%$ of the ASD-Only group had no regular periods, making determination of phase difficult. For the remaining participants where data was available, descriptively the groups had similar numbers within each phase, though the numbers were too low within each group for meaningful inferential statistics.

Finally, the sample sizes included here are relatively small with significant variability within each of the groups, particularly cortisol concentrations in those with ASD. The large degree of variability may make the detection of associations between cortisol concentrations and sleep parameters difficult. Studies with larger samples may be able to stratify patients based on sleep profiles, cortisol concentrations, and/or psychopathology symptoms to further elucidate these relationships.

\section{Conclusion}

Overall, this is the first study to systematically investigate relationships between cortisol, sleep, and psychopathology symptoms in a group of individuals with ASD. Despite ASD-only adults having evening cortisol levels that were suggestive of hypocortisolism, associations between evening physiological (cortisol) and subjective somatic arousal were associated with poor sleep in both groups of adults with ASD, and suggest a hyperarousal hypothesis of insomnia in ASD. These findings are consistent with the literature relating to individuals with burnout, which is attributed to chronic psychosocial stress. Individuals with higher burnout scores were shown to have a dampened response in relation to a psychosocial stressor in comparison to patients with lower burnout scores and controls, despite demonstrating a response to the stressor (Lennartsson, Sjörs, Währborg, Ljung, \& Jonsdottir, 2015). 
To confirm and extend on our findings, replication in larger samples of individuals with ASD is needed. In addition, exploration of other theories regarding poor sleep in ASD also warrant further research (e.g., circadian rhythm issues and behavioural problems). Given the potential for burnout to occur early in those with ASD, longitudinal studies beginning in early childhood, when poor sleep is first reported (Humphreys et al., 2014) would assist in further elucidating the relationships between sleep, anxiety and physiological arousal. Moreover, other physiological measures of arousal (e.g., heart rate, alpha amylase, respiratory rate) would assist in providing a more thorough understanding of CNS arousal and its role in ASD. 
Baker, E. Hyperarousal and Insomnia in ASD

\section{List of Abbreviations}

ADOS-2: Autism Diagnostic Observation Schedule-2 ${ }^{\text {nd }}$ Edition

AIC: Akaike Information Criterion

ANOVA: Analysis of variance

AQ: Autism Quotient

ASD: Autism Spectrum Disorder

BMI: Body Mass Index

CAR: Cortisol Awakening Response

CI: Confidence Interval

CNS: Central Nervous System

DLMO: Dim light melatonin onset

FDR: False Discovery Rate

FSIQ: Full scale IQ

M: Mean

PHQ-8: Patient Health Questionnaire-8

SAA-C: Sleep Anticipatory Anxiety-Cognitive

SAA-S: Sleep Anticipatory Anxiety-Somatic

SAAQ: Sleep Anticipatory Anxiety Questionnaire

SD: Standard deviation

SE\%: Sleep efficiency

SoL: Sleep onset latency

STAI-T: State Trait Anxiety Inventory-Trait subscale

TIB: Time in bed

TST: Total sleep time

This article is protected by copyright. All rights reserved. 
WAIS-IV: Wechsler Adult Intelligence Scale-Fourth Edition

WASI: Wechsler Abbreviated Scale of Intelligence

WASO: Wake after sleep onset

This article is protected by copyright. All rights reserved. 
Table 1

Group Comparisons for Age, FSIQ, AQ, BMI and ADOS using One-Way ANOVA

\begin{tabular}{|c|c|c|c|c|c|c|c|c|c|}
\hline & \multicolumn{2}{|c|}{$\begin{array}{c}\text { ASD-Only } \\
(n=16)\end{array}$} & \multicolumn{2}{|c|}{$\begin{array}{c}\text { ASD-Med } \\
(n=13)\end{array}$} & \multicolumn{2}{|c|}{$\begin{array}{l}\text { Control } \\
(n=29)\end{array}$} & \multirow[b]{2}{*}{$p$} & \multirow[b]{2}{*}{$F$} & \multirow[b]{2}{*}{$\eta^{2}$} \\
\hline & $M$ & $S D$ & $M$ & $S D$ & $M$ & $S D$ & & & \\
\hline Age (yrs) & 33.93 & 6.53 & 33.55 & 6.50 & 30.99 & 5.25 & .208 & 1.617 & .06 \\
\hline FSIQ & 120.69 & 14.23 & 116.92 & 9.15 & 119.18 & 7.84 & .652 & $.435^{\mathrm{a}}$ & .02 \\
\hline BMI & 25.55 & 5.66 & 26.24 & 3.90 & 24.95 & 4.30 & .743 & .299 & .01 \\
\hline \multirow[t]{2}{*}{ AQ Score } & 37.38 & 7.96 & 36.38 & 8.97 & 10.59 & 4.00 & $<.001$ & $113.974^{\mathrm{a}}$ & .81 \\
\hline & $M$ & $S D$ & $M$ & $S D$ & $M$ & $S D$ & $p$ & $t$ & $\eta^{2}$ \\
\hline ADOS Score ${ }^{b}$ & 12.81 & 4.12 & 10.08 & 3.95 & - & - & .081 & -1.812 & .11 \\
\hline
\end{tabular}

FSIQ = Full Scale IQ; BMI = Body Mass Index; AQ = Autism Quotient; ADOS = Autism Diagnostic Observation Schedule; M = Mean; SD = Standard Deviation

Note. FSIQ: Control $n=28$; BMI: ASD-Only $n=15$; ASD-Med $n=10$; Control $n=28$

${ }^{\mathrm{a}}$ Welch correction; ${ }^{\mathrm{b}}$ Independent Samples $t$ test 
Table 2

Group Comparisons for Sleep Variables

\begin{tabular}{|c|c|c|c|c|c|c|c|c|c|}
\hline & $\begin{array}{l}\text { ASD-Only } \\
(n=14)\end{array}$ & $\begin{array}{l}\text { ASD-Med } \\
\quad(n=9)\end{array}$ & $\begin{array}{l}\text { Control } \\
(n=27)\end{array}$ & & & & $\begin{array}{l}\text { ASD-Only } v \text {. } \\
\text { ASD-Med }\end{array}$ & $\begin{array}{l}\text { ASD-Only } v . \\
\text { Control }\end{array}$ & $\begin{array}{l}\text { ASD-Med } v . \\
\text { Control }\end{array}$ \\
\hline & $M \pm S D$ & $M \pm S D$ & $M \pm S D$ & $F$ & $p$ & $\eta^{2}$ & $\begin{array}{c}p \\
(95 \% \mathrm{CI})\end{array}$ & $\begin{array}{c}p \\
(95 \% \mathrm{CI})\end{array}$ & $\begin{array}{c}p \\
(95 \% \mathrm{CI})\end{array}$ \\
\hline \multicolumn{10}{|l|}{ Sleep } \\
\hline $\mathrm{PSQI}^{\mathrm{a}}$ & $7.36 \pm 3.39$ & $9.00 \pm 4.42$ & $4.74 \pm 1.70$ & 6.852 & .008 & .28 & $\begin{array}{c}.374 \\
(-1.30,4.58)\end{array}$ & $\begin{array}{c}. \mathbf{0 2 0} \\
(0.35,4.88)\end{array}$ & $\begin{array}{c}. \mathbf{0 0 1} \\
(1.61,6.91)\end{array}$ \\
\hline \multirow[t]{2}{*}{$\mathrm{TST}^{\mathrm{a}}$} & $230.93 \pm 43.57$ & $260.44 \pm 92.45$ & $319.07 \pm 58.29$ & 10.06 & $<.001$ & .30 & $\begin{array}{c}.512 \\
(-34.72,93.75)\end{array}$ & $\begin{array}{c}<.001 \\
(-137.66,38.63)\end{array}$ & $\begin{array}{c}.046 \\
(-116.50,-0.76)\end{array}$ \\
\hline & $\begin{array}{c}M d \\
M \pm S D\end{array}$ & $\begin{array}{c}M d \\
M \pm S D\end{array}$ & $\begin{array}{c}M d \\
M \pm S D\end{array}$ & $\chi^{2}$ & $p$ & & $\begin{array}{l}p \\
(r)\end{array}$ & $\begin{array}{l}p \\
(r)\end{array}$ & $\begin{array}{c}p \\
(r)\end{array}$ \\
\hline $\mathrm{SoL}^{\mathrm{b}}$ & $\begin{array}{c}7.50 \\
10.36 \pm 29.21\end{array}$ & $\begin{array}{c}3.00 \\
9.78 \pm 11.29\end{array}$ & $\begin{array}{c}9.00 \\
10.41 \pm 35.04\end{array}$ & .43 & .806 & - & & & \\
\hline WASO $^{\mathrm{b}}$ & $\begin{array}{c}24.00 \\
29.21 \pm 28.25\end{array}$ & $\begin{array}{c}45.00 \\
44.89 \pm 25.31\end{array}$ & $\begin{array}{c}29.00 \\
35.04 \pm 17.86\end{array}$ & 4.07 & .131 & - & & & \\
\hline
\end{tabular}




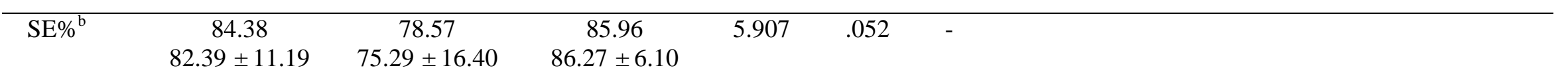

PSQI = Pittsburgh Sleep Quality Index; TST = Total sleep time; SoL = Sleep onset latency; WASO = Wake after sleep onset; SE\% = Sleep efficiency; M = Mean, SD = Standard Deviation; Md = Median

Note. Bolding indicates significance after FDR (corrected significance level $[p=.023]$ )

${ }^{\mathrm{a}}$ One-Way ANOVA with Tukey post-hoc tests; ${ }^{\mathrm{b}}$ Kruskal-Wallis tests. 
Table 3

Group Comparisons for Psychopathology Variables

\begin{tabular}{|c|c|c|c|c|c|c|c|c|c|}
\hline & $\begin{array}{l}\text { ASD-Only } \\
(n=14)\end{array}$ & $\begin{array}{l}\text { ASD-Med } \\
\quad(n=9)\end{array}$ & $\begin{array}{l}\text { Control } \\
(n=27)\end{array}$ & & & & $\begin{array}{c}\text { ASD-Only } v . \\
\text { ASD-Med }\end{array}$ & $\begin{array}{c}\text { ASD-Only } v \text {. } \\
\text { Control }\end{array}$ & $\begin{array}{l}\text { ASD-Med } v \text {. } \\
\text { Control }\end{array}$ \\
\hline & $M \pm S D$ & $M \pm S D$ & $M \pm S D$ & $F$ & $p$ & $\eta^{2}$ & $\begin{array}{c}p \\
(95 \% \mathrm{CI})\end{array}$ & $\begin{array}{c}p \\
(95 \% \mathrm{CI})\end{array}$ & $\begin{array}{c}p \\
(95 \% \mathrm{CI})\end{array}$ \\
\hline \multicolumn{10}{|l|}{ Psychopathology } \\
\hline Trait Anxiety $^{\mathrm{a}}$ & $53.36 \pm 14.44$ & $57.44 \pm 12.19$ & $36.26 \pm 10.03$ & 15.997 & $<.001$ & .41 & $\begin{array}{c}.697 \\
(-8.08,16.26)\end{array}$ & $\begin{array}{c}<.001 \\
(7.72,26.48)\end{array}$ & $\begin{array}{c}<.001 \\
(10.22,32.15)\end{array}$ \\
\hline \multirow[t]{2}{*}{ Cognitive Arousal $^{\mathrm{a}}$} & $9.00 \pm 4.44$ & $9.33 \pm 3.00$ & $4.52 \pm 3.69$ & 9.114 & $<.001$ & .28 & $\begin{array}{c}.977 \\
(-3.61,4.27)\end{array}$ & $\begin{array}{c}.002 \\
(1.44,7.52)\end{array}$ & $\begin{array}{c}.005 \\
(1.26,8.37)\end{array}$ \\
\hline & $\begin{array}{c}M d \\
M \pm S D\end{array}$ & $\begin{array}{c}M d \\
M \pm S D\end{array}$ & $\begin{array}{c}M d \\
M \pm S D\end{array}$ & $\chi^{2}$ & $p$ & & $\begin{array}{l}p \\
(r)\end{array}$ & $\begin{array}{c}p \\
(r)\end{array}$ & $\begin{array}{l}p \\
(r)\end{array}$ \\
\hline Somatic Arousal $^{b}$ & $\begin{array}{c}4.00 \\
4.00 \pm 2.99\end{array}$ & $\begin{array}{c}5.00 \\
5.00 \pm 1.00\end{array}$ & $\begin{array}{c}1.00 \\
1.78 \pm 2.31\end{array}$ & 14.294 & .001 & - & $\begin{array}{l}.199 \\
(.28)\end{array}$ & $\begin{array}{l}\mathbf{. 0 1 7} \\
(.37)\end{array}$ & $\begin{array}{l}<.001 \\
(.59)\end{array}$ \\
\hline Depression $^{\mathrm{b}}$ & $\begin{array}{c}7.50 \\
8.79 \pm 5.26\end{array}$ & $\begin{array}{c}11.00 \\
12.67 \pm 6.60\end{array}$ & $\begin{array}{c}2.00 \\
2.74 \pm 2.60\end{array}$ & 24.934 & $<.001$ & - & $\begin{array}{l}.163 \\
(.29)\end{array}$ & $\begin{array}{l}<.001 \\
(.59)\end{array}$ & $\begin{array}{l}<.001 \\
(.67)\end{array}$ \\
\hline
\end{tabular}

$\mathrm{M}=$ Mean, $\mathrm{SD}=$ Standard Deviation; $\mathrm{Md}=$ Median

Note. Bolding indicates significance after FDR (corrected significance level [ $p=.0375]$ )

This article is protected by copyright. All rights reserved. 
${ }^{a}$ One-Way ANOVA with Tukey post-hoc tests; ${ }^{\text {b }}$ Kruskal-Wallis tests with follow-up Mann Whitney $U$ tests. 
Table 4

Wake After Sleep Onset (WASO) Association with Evening Cortisol, Somatic Arousal and Depression

\section{Model 1}

Variable

Estimate

$95 \%$ CI

$p$

\section{Cortisol}

$\begin{array}{lccc}\text { ASD-Only } & 10.859 & (3.871,17.847) & .003 \\ \text { ASD-Med } & 1.294 & (-2.652,5.240) & .511 \\ \text { Controls } & -1.872 & (-5.726,1.982) & .332\end{array}$

Somatic Arousal

$\begin{array}{lccc}\text { ASD-Only } & 1.928 & (-3.095,6.952) & .442 \\ \text { ASD-Med } & -24.723 & (-40.733,-8.713) & .003 \\ \text { Controls } & 0.011 & (-3.396,3.417) & .995\end{array}$

Depression

$\begin{array}{lccc}\text { ASD-Only } & -0.830 & (-3.745,2.085) & .568 \\ \text { ASD-Med } & 2.977 & (0.747,5.207) & \mathbf{. 0 1 0} \\ \text { Controls } & 0.189 & (-2.812,3.189) & .899\end{array}$

Note. Bolding indicates significance after FDR (corrected significance level [ $p=.017]$ ); Estimate $=$ the mean change in WASO per unit of cortisol, somatic arousal, and depression. 
Baker, E. Hyperarousal and Insomnia in ASD

Table 5

Sleep Efficiency (SE\%) Association with Evening Cortisol, Somatic Arousal and Anxiety

\section{Model 1}

Variable

Estimate

$95 \%$ CI

$p$

\section{Cortisol}

$\begin{array}{lccc}\text { ASD-Only } & -3.098 & (-5.343,-0.854) & \mathbf{. 0 0 8} \\ \text { ASD-Med } & -4.080 & (-5.491,-2.669) & <.001 \\ \text { Controls } & 0.267 & (-1.100,1.633) & .695\end{array}$

\section{Somatic Arousal}

$\begin{array}{lccc}\text { ASD-Only } & -1.859 & (-3.222,-0.496) & .009 \\ \text { ASD-Med } & 1.161 & (-4.281,6.602) & .669 \\ \text { Controls } & -.233 & (-1.458,0.992) & .702\end{array}$

\section{Trait Anxiety}
All groups
$-0.152$
$(-0.345,0.041)$
.120

Note. Bolding indicates significance after FDR (corrected significance level [ $p=.025]$ ); Estimate $=$ the mean change in SE\% per unit of cortisol, somatic arousal, and trait anxiety. 
Baker, E. Hyperarousal and Insomnia in ASD

Table 6

Total Sleep Time (TST) Association with Anxiety, Somatic Arousal and Depression

\section{Model 1}

Variable

Estimate

95\% CI

$p$

\section{Cortisol}

$\begin{array}{lccc}\text { ASD-Only } & 4.558 & (-14.752,23.867) & .636 \\ \text { ASD-Med } & -17.382 & (-28.303,-6.461) & .003 \\ \text { Controls } & -5.053 & (-16.431,6.325) & .375\end{array}$

Trait Anxiety

$\begin{array}{lccc}\text { ASD-Only } & -0.192 & (-2.678,2.293) & .876 \\ \text { ASD-Med } & -2.857 & (-6.93,1.215) & .164 \\ \text { Controls } & 1.261 & (-1.17,3.691) & .301\end{array}$

\section{Somatic Arousal}
All groups
$-11.200$
$(-19.678,-2.722)$
.011

\section{Depression}
All groups
4.707
$(-0.774,10.188)$
.090

Note. Bolding indicates significance after FDR (corrected significance level [ $p=.0125]$ ); Estimate $=$ the mean change in TST per unit of cortisol, trait anxiety, somatic arousal, and depression. 


\section{Figure Legends}

Figure 1. Saliva Collection Protocol

Figure 2. Evening Cortisol Concentrations for the ASD-Only, ASD-Med and Control groups. Error bars indicate standard errors.

Figure 3. Morning Cortisol Concentrations for the ASD-Only, ASD-Med and Control groups. Error bars indicate standard errors.

\section{References}

American Psychiatric Association. (2000). Diagnostic and statistical manual of mental disorders text revision (4th ed.). Washington, D.C.: American Psychiatric Association.

Backhaus, J., Junghanns, K., Broocks, A., Riemann, D., \& Hohagen, F. (2002). Test-retest reliability and validity of the Pittsburgh Sleep Quality Index in primary insomnia. $J$ Psychosom Res, 53(3), 737-740.

Backhaus, J., Junghanns, K., \& Hohagen, F. (2004). Sleep disturbances are correlated with decreased morning awakening salivary cortisol. Psychoneuroendocrinology, 29(9), 1184-1191.

Baker, E. K., \& Richdale, A. L. (2015). Sleep Patterns in Adults with a Diagnosis of HighFunctioning Autism Spectrum Disorder. Sleep.

Baker, E. K., Richdale, A. L., Hazi, A., \& Prendergast, L. A. (2017). Assessing the Dim Light Melatonin Onset in Adults with Autism Spectrum Disorder and No Comorbid Intellectual Disability. J Autism Dev Disord, 47(7), 2120-2137. doi:10.1007/s10803$017-3122-4$

This article is protected by copyright. All rights reserved. 
Baron-Cohen, S., Wheelwright, S., Skinner, R., Martin, J., \& Clubley, E. (2001). The Autism-Spectrum Quotient (AQ): Evidence from Asperger Syndrome/HighFunctioning Autism, Malesand Females, Scientists and Mathematicians. Journal of Autism and Developmental Disorders, 31(1), 5-17. doi:10.1023/a:1005653411471

Benca, R. M., Obermeyer, W. H., Thisted, R. A., \& Gillin, J. C. (1992). Sleep and psychiatric disorders. A meta-analysis. Archives of General Psychiatry, 49(8), 651-668; discussion 669-670.

Benjamini, Y., \& Hochberg, Y. (1995). Controlling the False Discovery Rate: A Practical and Powerful Approach to Multiple Testing. Journal of the Royal Statistical Society. Series B (Methodological), 57(1), 289-300. doi:10.2307/2346101

Benloucif, S., Burgess, H. J., Klerman, E. B., Lewy, A. J., Middleton, B., Murphy, P. J., . . . Revell, V. L. (2008). Measuring melatonin in humans. J Clin Sleep Med, 4(1), 66-69.

Benowitz, N. L. (1990). Clinical Pharmacology of Caffeine. Annual Review of Medicine, 41(1), 277-288. doi:doi:10.1146/annurev.me.41.020190.001425

Bitsika, V., Sharpley, C. F., Sweeney, J. A., \& McFarlane, J. R. (2014). HPA and SAM axis responses as correlates of self- vs parental ratings of anxiety in boys with an Autistic Disorder. Physiology \& Behavior, 127, 1-7. doi:10.1016/j.physbeh.2013.12.011

Bonnet, M. H., \& Arand, D. L. (1997). Hyperarousal and insomnia. Sleep Med Rev, 1(2), 97108.

Bonnet, M. H., \& Arand, D. L. (2003). Situational insomnia: consistency, predictors, and outcomes. Sleep, 26(8), 1029-1036.

This article is protected by copyright. All rights reserved. 
Bootzin, R., Shoham, V., \& Kuo, T. (1994). Sleep anticipatory anxiety questionnaire: A measure of anxiety about sleep. Sleep Research, 23, 188.

Broman, J. E., \& Hetta, J. (1994). Electrodermal activity in patients with persistent insomnia. J Sleep Res, 3(3), 165-170.

Buck, T., Viskochil, J., Farley, M., Coon, H., McMahon, W., Morgan, J., \& Bilder, D. (2014). Psychiatric Comorbidity and Medication Use in Adults with Autism Spectrum Disorder. Journal of Autism and Developmental Disorders, 44(12), 3063-3071. doi:10.1007/s10803-014-2170-2

Buysse, D. J., Reynolds III, C. F., Monk, T. H., Berman, S. R., \& Kupfer, D. J. (1989). The Pittsburgh sleep quality index: A new instrument for psychiatric practice and research. Psychiatry Research, 28(2), 193-213.

Cascade, E., Kalali, A. H., \& Kennedy, S. H. (2009). Real-World Data on SSRI Antidepressant Side Effects. Psychiatry (Edgmont), 6(2), 16-18.

Chicoine, M., Limoges, E., Chevrier, E., Lupien, S., Mottron, L., \& Godbout, R. (2013). Is cortisol associated with poor sleep in autism? A laboratory study in high functioning adults. Paper presented at the 5th World Congress on Sleep Medicine, Valencia, Spain.

Chida, Y., \& Steptoe, A. (2009). Cortisol awakening response and psychosocial factors: a systematic review and meta-analysis. Biological Psychology, 80(3), 265-278. doi:10.1016/j.biopsycho.2008.10.004

This article is protected by copyright. All rights reserved. 
Corbett, B. A., Mendoza, S., Wegelin, J. A., Carmean, V., \& Levine, S. (2008). Variable cortisol circadian rhythms in children with autism and anticipatory stress. Journal of Psychiatry and Neuroscience, 33(3), 227-234.

Crawford, J., Cayley, C., Lovibond, P. F., Wilson, P. H., \& Hartley, C. (2011). Percentile Norms and Accompanying Interval Estimates from an Australian General Adult Population Sample for Self-Report Mood Scales (BAI, BDI, CRSD, CES-D, DASS, DASS-21, STAI-X, STAI-Y, SRDS, and SRAS). Australian Psychologist, 46(1), 314. doi:10.1111/j.1742-9544.2010.00003.x

Dahl, R. E., Ryan, N. D., Matty, M. K., Birmaher, B., al-Shabbout, M., Williamson, D. E., \& Kupfer, D. J. (1996). Sleep onset abnormalities in depressed adolescents. Biological Psychiatry, 39(6), 400-410.

Dieleman, G. C., Huizink, A. C., Tulen, J. H., Utens, E. M., Creemers, H. E., van der Ende, J., \& Verhulst, F. C. (2015). Alterations in HPA-axis and autonomic nervous system functioning in childhood anxiety disorders point to a chronic stress hypothesis. Psychoneuroendocrinology, 51, 135-150. doi:10.1016/j.psyneuen.2014.09.002

Edmiston, E. K., Blain, S. D., \& Corbett, B. A. (2017). Salivary cortisol and behavioral response to social evaluative threat in adolescents with autism spectrum disorder. Autism Research, 10(2), 346-358. doi:10.1002/aur.1660

Fahrmeir, L., Kneib, T., Lang, S., \& Marx, B. (2013). Regression: Models, Methods and Applications. Berlin, Germany: Springer-Verlag Berlin Heidelberg.

Feder, A., Coplan, J. D., Goetz, R. R., Mathew, S. J., Pine, D. S., Dahl, R. E., . . Weissman, M. M. (2004). Twenty-four-hour cortisol secretion patterns in prepubertal children 
with anxiety or depressive disorders. Biol Psychiatry, 56(3), 198-204. doi:10.1016/j.biopsych.2004.05.005

Forbes, E. E., Williamson, D. E., Ryan, N. D., Birmaher, B., Axelson, D. A., \& Dahl, R. E. (2006). Peri-sleep-onset cortisol levels in children and adolescents with affective disorders. Biological Psychiatry, 59(1), 24-30. doi:S0006-3223(05)00708-0 [pii] 10.1016/j.biopsych.2005.06.002 [doi]

Goldman, S. E., Alder, M. L., Burgess, H. J., Corbett, B. A., Hundley, R., Wofford, D., .. . Malow, B. A. (2017). Characterizing Sleep in Adolescents and Adults with Autism Spectrum Disorders. J Autism Dev Disord, 47(6), 1682-1695. doi:10.1007/s10803017-3089-1

Granger, D. A., Hibel, L. C., Fortunato, C. K., \& Kapelewski, C. H. (2009). Medication effects on salivary cortisol: tactics and strategy to minimize impact in behavioral and developmental science. Psychoneuroendocrinology, 34(10), 1437-1448. doi:10.1016/j.psyneuen.2009.06.017

Heim, C., Ehlert, U., \& Hellhammer, D. H. (2000). The potential role of hypocortisolism in the pathophysiology of stress-related bodily disorders. Psychoneuroendocrinology, 25(1), 1-35.

Hoehn-Saric, R., \& McLeod, D. R. (2000). Anxiety and arousal: physiological changes and their perception. Journal of Affective Disorders, 61(3), 217-224. doi:http://dx.doi.org/10.1016/S0165-0327(00)00339-6

Hollocks, M. J., Howlin, P., Papadopoulos, A. S., Khondoker, M., \& Simonoff, E. (2014). Differences in HPA-axis and heart rate responsiveness to psychosocial stress in 
children with autism spectrum disorders with and without co-morbid anxiety. Psychoneuroendocrinology, 46, 32-45. doi:10.1016/j.psyneuen.2014.04.004

Hollway, J. A., Aman, M. G., \& Butter, E. (2013). Correlates and risk markers for sleep disturbance in participants of the Autism Treatment Network. Journal of Autism and Developmental Disorders, 43(12), 2830-2843. doi:10.1007/s10803-013-1830-y

Humphreys, J. S., Gringras, P., Blair, P. S., Scott, N., Henderson, J., Fleming, P. J., \& Emond, A. M. (2014). Sleep patterns in children with autistic spectrum disorders: a prospective cohort study. Arch Dis Child, 99(2), 114-118. doi:10.1136/archdischild2013-304083

Jackowska, M., Ronaldson, A., Brown, J., \& Steptoe, A. (2016). Biological and psychological correlates of self-reported and objective sleep measures. Journal of Psychosomatic Research, 84, 52-55. doi:10.1016/j.jpsychores.2016.03.017

Jung, C. M., Khalsa, S. B. S., Scheer, F. A. J. L., Cajochen, C., Lockley, S. W., Czeisler, C. A., \& Wright, K. P. (2010). Acute Effects of Bright Light Exposure on Cortisol Levels. Journal of Biological Rhythms, 25(3), 208-216. doi:10.1177/0748730410368413

Karlamangla, A. S., Friedman, E. M., Seeman, T. E., Stawksi, R. S., \& Almeida, D. M. (2013). Daytime trajectories of cortisol: demographic and socioeconomic differences-findings from the National Study of Daily Experiences. Psychoneuroendocrinology, 38(11), 2585-2597. doi:10.1016/j.psyneuen.2013.06.010

Kroenke, K., Strine, T. W., Spitzer, R. L., Williams, J. B. W., Berry, J. T., \& Mokdad, A. H. (2009). The PHQ-8 as a measure of current depression in the general population. 
Journal of Affective Disorders, 114(1-3), 163-173. doi:http://dx.doi.org/10.1016/j.jad.2008.06.026

Kunz-Ebrecht, S. R., Kirschbaum, C., Marmot, M., \& Steptoe, A. (2004). Differences in cortisol awakening response on work days and weekends in women and men from the Whitehall II cohort. Psychoneuroendocrinology, 29(4), 516-528.

Kushki, A., Brian, J., Dupuis, A., \& Anagnostou, E. (2014). Functional autonomic nervous system profile in children with autism spectrum disorder. Molecular Autism, 5, 39. doi:10.1186/2040-2392-5-39

Kushki, A., Drumm, E., Pla Mobarak, M., Tanel, N., Dupuis, A., Chau, T., \& Anagnostou, E. (2013). Investigating the autonomic nervous system response to anxiety in children with autism spectrum disorders. PLoS One, 8(4), e59730. doi:10.1371/journal.pone.0059730

Lennartsson, A., Sjörs, A., Währborg, P., Ljung, T., \& Jonsdottir, I. H. (2015). Burnout and Hypocortisolism - A Matter of Severity? A Study on ACTH and Cortisol Responses to Acute Psychosocial Stress. Frontiers in psychiatry, 6, 8-8. doi:10.3389/fpsyt.2015.00008

Limoges, É., Mottron, L., Bolduc, C., Berthiaume, C., \& Godbout, R. (2005). Atypical sleep architecture and the autism phenotype. Brain, 128, 1049-1061. doi:awh425 [pii] 10.1093/brain/awh425

Lord, C., Rutter, M., DiLavore, P. C., Risi, S., Gotham, K., \& Bishop, S. L. (2012). Autism Diagnostic Observation Schedule (2nd ed.). Los Angeles, CA: Western Psychological Services.

This article is protected by copyright. All rights reserved. 
Masharani, U., Shiboski, S., Eisner, M. D., Katz, P. P., Janson, S. L., Granger, D. A., \& Blanc, P. D. (2005). Impact of exogenous glucocorticoid use on salivary cortisol measurements among adults with asthma and rhinitis. Psychoneuroendocrinology, 30(8), 744-752. doi:10.1016/j.psyneuen.2005.03.003

Mayes, S. D., \& Calhoun, S. L. (2009). Variables related to sleep problems in children with autism. Research in Autism Spectrum Disorders, 3(4), 931-941.

Mazurek, M. O., \& F., P. G. (2015). Sleep problems in children with autism spectrum disorder: Examining the contributions of sensory over-responsivity and anxiety. Sleep Medicine, 16, 270-279. doi:10.1016/j.sleep.2014.11.006

Monroe, L. J. (1967). Psychological and physiological differences between good and poor sleepers. J Abnorm Psychol, 72(3), 255-264.

Muscatello, R. A., \& Corbett, B. A. (2018). Comparing the effects of age, pubertal development, and symptom profile on cortisol rhythm in children and adolescents with autism spectrum disorder. Autism Research, 11(1), 110-120. doi:doi:10.1002/aur.1879

Richdale, A. L., Baker, E. K., Short, M., \& Gradisar, M. (2014). The role of insomnia, presleep arousal and psychopathology symptoms in daytime impairment in adolescents with high-functioning autism spectrum disorder. Sleep Medicine, 15(9), 1082-1088. doi:10.1016/j.sleep.2014.05.005

Richdale, A. L., \& Prior, M. R. (1992). Urinary cortisol circadian rhythm in a group of highfunctioning children with autism. Journal of Autism and Developmental Disorders, 22(3), 433-447.

This article is protected by copyright. All rights reserved. 
Richdale, A. L., \& Schreck, K. A. (2009). Sleep problems in autism spectrum disorders: Prevalence, nature \& possible biopsychosocial aetiologies. Sleep Medicine Reviews, 13(6), 403-411. doi:10.1016/j.smrv.2009.02.003.

Rodenbeck, A., Huether, G., Ruther, E., \& Hajak, G. (2002). Interactions between evening and nocturnal cortisol secretion and sleep parameters in patients with severe chronic primary insomnia. Neuroscience Letters, 324(2), 159-163. doi:S0304394002001921 [pii]

Sharpley, C. F., Bitsika, V., Agnew, L. L., \& Andronicos, N. M. (2017). Is daily replication necessary when sampling cortisol concentrations in association studies of children with autism spectrum disorder? A systematic review and discussion paper Reviews in the Neurosciences (Vol. 28, pp. 103).

Shin, Y. S., Liu, J. N., Kim, J. H., Nam, Y. H., Choi, G. S., Park, H. S., . . . Allergic Diseases Study, G. (2014). The impact of asthma control on salivary cortisol level in adult asthmatics. Allergy Asthma \& Immunology Research, 6(5), 463-466. doi:10.4168/aair.2014.6.5.463

Simon, D. M., \& Corbett, B. A. (2013). Examining associations between anxiety and cortisol in high functioning male children with autism. Journal of Neurodevelopmental Disorders, 5(1), 32. doi:10.1186/1866-1955-5-32

Spence, S. H. (1998). A measure of anxiety symptoms among children. Behaviour Research and Therapy, 36(5), 545-566.

Spielberger, C. D., Gorsuch, R. L., Lushene, R., Vagg, P. R., \& Jacobs, G. A. (1983). Manual for the State-Trait Anxiety Inventory. Palo Alto, CA: Consulting Psychologists Press. 
Stalder, T., Kirschbaum, C., Kudielka, B. M., Adam, E. K., Pruessner, J. C., Wüst, S., .. . Clow, A. (2016). Assessment of the cortisol awakening response: Expert consensus guidelines. Psychoneuroendocrinology, 63, 414-432. doi:http://dx.doi.org/10.1016/j.psyneuen.2015.10.010

Strang, J. F., Kenworthy, L., Daniolos, P., Case, L., Wills, M. C., Martin, A., \& Wallace, G. L. (2012). Depression and anxiety symptoms in children and adolescents with autism spectrum disorders without intellectual disability. Research in Autism Spectrum Disorders, 6(1), 406-412. doi:http://dx.doi.org/10.1016/j.rasd.2011.06.015

Tomarken, A. J., Han, G. T., \& Corbett, B. A. (2015). Temporal patterns, heterogeneity, and stability of diurnal cortisol rhythms in children with autism spectrum disorder. Psychoneuroendocrinology, 62, 217-226. doi:10.1016/j.psyneuen.2015.08.016

Tordjman, S., Anderson, G. M., Kermarrec, S., Bonnot, O., Geoffray, M., Brailly-Tabard, S., ... Touitou, Y. (2014). Altered circadian patterns of salivary cortisol in lowfunctioning children and adolescents with autism. Psychoneuroendocrinology, 50, 227-245. doi:http://dx.doi.org/10.1016/j.psyneuen.2014.08.010

Vgontzas, A. N., Bixler, E. O., Lin, H. M., Prolo, P., Mastorakos, G., Vela-Bueno, A., .. . Chrousos, G. P. (2001). Chronic insomnia is associated with nyctohemeral activation of the hypothalamic-pituitary-adrenal axis: clinical implications. The Journal of Clinical Endocrinology \& Metabolism, 86(8), 3787-3794. doi:10.1210/jcem.86.8.7778

Wechsler, D. (1999). WASI Administration and Scoring Manual. San Antonio, TX: The Psychological Corporation. 
Wechsler, D. (2008). WAIS-IV Administration and Scoring Manual. San Antonio, TX: The Psychological Corporation.

Weinkauf, M. (2012). BenjaminiHochberg.xlsx.

West, C. H. K., Ritchie, J. C., Boss-Williams, K. A., \& Weiss, J. M. (2009). Antidepressant drugs with differing pharmacological actions decrease activity of locus coeruleus neurons. The international journal of neuropsychopharmacology, 12(5), 627-641. doi:10.1017/S1461145708009474

Wiggs, L., \& Stores, G. (2004). Sleep patterns and sleep disorders in children with autistic spectrum disorders: insights using parent report and actigraphy. Developmental Medicine \& Child Neurology, 46(06), 372-380. doi:doi:10.1017/S0012162204000611

Woodbury-Smith, M. R., Robinson, J., Wheelwright, S., \& Baron-Cohen, S. (2005). Screening adults for Asperger Syndrome using the AQ: a preliminary study of its diagnostic validity in clinical practice. Journal of Autism and Developmental Disorders, 35(3), 331-335.

This article is protected by copyright. All rights reserved. 


\section{University Library}

\section{- M M N E R VA A gateway to Melbourne's research publications}

Minerva Access is the Institutional Repository of The University of Melbourne

Author/s:

Baker, EK;Richdale, AL;Hazi, A;Prendergast, LA

Title:

Assessing a hyperarousal hypothesis of insomnia in adults with autism spectrum disorder

Date:

2019-06-01

Citation:

Baker, E. K., Richdale, A. L., Hazi, A. \& Prendergast, L. A. (2019). Assessing a hyperarousal hypothesis of insomnia in adults with autism spectrum disorder. AUTISM RESEARCH, 12 (6), pp.897-910. https://doi.org/10.1002/aur.2094.

Persistent Link:

http://hdl.handle.net/11343/285632 